\title{
Approximation on a class of Szász-Mirakyan operators via second kind of beta operators
}

\author{
M. Nasiruzzaman ${ }^{1 *}$, Nadeem Rao², Anshul Srivastava ${ }^{3}$ and Ravi Kumar ${ }^{4}$
}

\section{${ }^{*}$ Correspondence:}

nasir3489@gmail.com

'Department of Mathematics,

Faculty of Science, University of

Tabuk, Tabuk, Saudi Arabia

Full list of author information is

available at the end of the article

\begin{abstract}
In the present article, we construct a new sequence of positive linear operators via Dunkl analogue of modified Szász-Durrmeyer operators. We study the moments and basic results. Further, we investigate the pointwise approximation and uniform approximation results in various functional spaces for these sequences of positive linear operators. Finally, we prove the global approximation and A-statistical convergence results for these operators.
\end{abstract}

MSC: Primary 41A25; 41A36; secondary 33C45

Keywords: Szász-Mirakyan operators; Modulus of continuity; Rate of convergence; Dunkl analogue

\section{Introduction}

In recent past, the Szász-Mirakjan operators and Szász-Durrmeyer type operators have been intensively investigated in various functional spaces to approximate the continuous functions and Lebesgue measurable functions respectively. Mazhar and Totik [17] gave an integral modification of Szász-Mirakyan [37] operators to study the Lebesgue measurable functions as follows:

$$
S_{n}^{*}(g ; u)=n e^{-n u} \sum_{k=0}^{\infty} \frac{(n u)^{k}}{k !} \int_{0}^{\infty} e^{-n t} \frac{(n t)^{k}}{k !} g(t) d t, \quad u \geq 0, n \in \mathbb{N}=\{1,2,3, \ldots\} .
$$

Researchers have obtained several approximations of Szász-Mirakyan type operators via Dunkl generalization; for instance, see [6, 18, 26, 28, 29, 32, 39]. Related to these results, more approximation results have been studied in different functional spaces (see $[1,2,4$, $5,14,38]$ and [3, 16, 27, 31]). Sucu [36] introduced Szász-Mirakyan type operators for $k \in \mathbb{N}_{0}=\mathbb{N} \cup\{0\}$ as follows:

$$
S_{n}(g ; u):=\frac{1}{e_{i}(n u)} \sum_{k=0}^{\infty} \frac{(n u)^{k}}{\gamma_{i}(k)} g\left(\frac{k+2 i \theta_{k}}{n}\right) \text {, }
$$

(c) The Author(s) 2020. This article is licensed under a Creative Commons Attribution 4.0 International License, which permits use sharing, adaptation, distribution and reproduction in any medium or format, as long as you give appropriate credit to the original author(s) and the source, provide a link to the Creative Commons licence, and indicate if changes were made. The images or other third party material in this article are included in the article's Creative Commons licence, unless indicated otherwise in a credit line to the material. If material is not included in the article's Creative Commons licence and your intended use is not permitted by statutory regulation or exceeds the permitted use, you will need to obtain permission directly from the copyright holder. To view a copy of this licence, visit http://creativecommons.org/licenses/by/4.0/. 
using generalized exponential function [33] given by

$$
e_{i}(t)=\sum_{j=0}^{\infty} \frac{t^{j}}{\gamma_{i}(j)}, \quad t \in[0, \infty),
$$

where the coefficients $\gamma_{i}$ are defined as follows:

$$
\gamma_{i}(2 j)=\frac{2^{2 j} j ! \Gamma\left(j+i+\frac{1}{2}\right)}{\Gamma\left(i+\frac{1}{2}\right)} \quad \text { and } \quad \gamma_{i}(2 j+1)=2^{2 j+1} j ! \frac{\Gamma\left(j+i+\frac{3}{2}\right)}{\Gamma\left(i+\frac{1}{2}\right)} \text {. }
$$

Here, $\Gamma$ is the gamma function and $i>-\frac{1}{2}$. In [36] the pointwise and uniform approximation results for the operators defined by (1.2) are obtained. Several extensions of the Szász-Mirakyan operators defined by (1.2) and their approximation results are studied by the authors viz İçöz et al. [11, 12]. In [40], we have constructed Szász-Durrmeyer type operators to approximate the Lebesgue measurable functions as follows:

$$
D_{n}(g ; u)=\frac{1}{e_{i}(n u)} \sum_{j=0}^{\infty} \frac{(n u)^{j}}{\gamma_{i}(j)} \frac{n^{j+2 i \theta_{j}+\lambda+1}}{\Gamma\left(j+2 i \theta_{j}+\lambda+1\right)} \int_{0}^{\infty} t^{j+2 i \theta_{j}+\lambda} e^{-n t} g(t) d t
$$

where $\Gamma(t)=\int_{0}^{\infty} u^{t} e^{-u} d u$ is the gamma function, $\lambda \geq 0$, and

$$
\theta_{j}= \begin{cases}0, & j \in 2 \mathbb{N}, \\ 1, & j \in 2 \mathbb{N}+1\end{cases}
$$

In order to obtain a better rate of convergence in comparison to (1.4), in this paper we introduce a modification with two nonnegative shifted nodes as follows:

$$
M_{n}^{\alpha, \beta}(g ; u)=\sum_{k=0}^{\infty} v_{n}(u ; k) \int_{0}^{\infty} t^{k+2 i \theta_{k}+\lambda} e^{-n t} g\left(\frac{n t+\alpha}{n+\beta}\right) d t,
$$

where $v_{n}(u ; k)=\frac{1}{e_{i}(n u)} \frac{(n u)^{k}}{\gamma_{i}(k)} \frac{n^{k+2 i \theta_{k}+\lambda+1}}{\Gamma\left(k+2 i \theta_{k}+\lambda+1\right)}, 0 \leq \alpha \leq \beta$, and $f \in C[0, \infty)$ whenever the above series converges. One can notice that (i) for $i=\lambda=\alpha=\beta=0$, the sequence of operators introduced in (1.5) reduces to the operators defined in (1.1) and (ii) for $\alpha=\beta=0$, the sequence of positive linear operators defined in (1.5) reduces to the operators defined in [40].

In the subsequent sections, we deduce some basic results, direct approximation results. Further, global approximation results are studied in [19-21, 24, 25, 35].

\section{Preliminary results}

For $i>-\frac{1}{2}, u \geq 0$, and $n \in \mathbb{N}$, we denote

$$
E_{n}(u)=\frac{e_{i}(-n u)}{e_{i}(n u)} .
$$

Lemma 2.1 ([40]) For the operators given by (1.4), we have

$$
D_{n}(1 ; u)=1 \text {, }
$$




$$
\begin{aligned}
D_{n}(t ; u)= & u+\frac{\lambda+1}{n}, \\
D_{n}\left(t^{2} ; u\right)= & u^{2}+\left(4+2 \lambda+2 i E_{n}(u)\right) \frac{u}{n}+\frac{(\lambda+1)(\lambda+2)}{n^{2}}, \\
D_{n}\left(t^{3} ; u\right)= & u^{3}+\left(9+3 \lambda-2 i E_{n}(u)\right) \frac{u^{2}}{n}+\left(18+3 \lambda(\lambda+5)+4 i^{2}\right. \\
& \left.+2 i(8+3 \lambda) E_{n}(u)\right) \frac{u}{n^{2}}+\frac{\lambda^{3}+6 \lambda^{2}+11 \lambda+6}{n^{3}}, \\
D_{n}\left(t^{4} ; u\right)= & u^{4}+\mathcal{O}\left(\frac{1}{n}\right) \text { for each compact subset of }[0, \infty) .
\end{aligned}
$$

Lemma 2.2 For operators (1.5), we have

$$
M_{n}^{\alpha, \beta}\left(t^{r} ; u\right)=\sum_{i=0}^{r}\left(\begin{array}{l}
r \\
i
\end{array}\right) \frac{n^{i} \alpha^{r-i}}{(n+\beta)^{r}} D_{n}\left(t^{i} ; u\right), \quad r \in \mathbb{N} .
$$

Proof From (1.5), we get

$$
\begin{aligned}
M_{n}^{\alpha, \beta}\left(t^{r} ; u\right) & =\sum_{k=0}^{\infty} v_{n}(u ; t) \int_{0}^{\infty} t^{k+2 i \theta_{k}+\lambda} e^{-n t}\left(\frac{n t+\alpha}{n+\beta}-u\right)^{r} \\
& =\sum_{j=0}^{r}\left(\begin{array}{l}
r \\
j
\end{array}\right) \frac{n^{j} \alpha^{r-j}}{(n+\beta)^{r}} \sum_{k=0}^{\infty} v_{n}(u ; t) \int_{0}^{\infty} t^{k+2 i \theta_{k}+\lambda} e^{-n t} t^{j} d t \\
& =\sum_{j=0}^{r}\left(\begin{array}{l}
r \\
j
\end{array}\right) \frac{n^{j} \alpha^{r-j}}{(n+\beta)^{r}} D_{n}\left(t^{j} ; u\right) .
\end{aligned}
$$

Lemma 2.3 For $r \in \mathbb{N}$, we have the following relation:

$$
M_{n}^{\alpha, \beta}\left((t-u)^{r} ; u\right)=\sum_{i=0}^{r}\left(\begin{array}{l}
r \\
i
\end{array}\right)(-u)^{r-i} M_{n}^{\alpha, \beta}\left(t^{i} ; u\right)
$$

where $M_{n}^{\alpha, \beta}$ is defined in (1.5).

Proof From relation (1.5), we get

$$
\begin{aligned}
M_{n}^{\alpha, \beta}\left((t-u)^{r} ; u\right) & =\sum_{k=0}^{\infty} v_{n}(u ; k) \int_{0}^{\infty} t^{k+2 i \theta_{k}+\lambda} e^{-n t}\left(\frac{n t+\alpha}{n+\beta}-u\right)^{r} d t \\
& =\sum_{j=0}^{r}\left(\begin{array}{l}
r \\
j
\end{array}\right)(-u)^{r-j} M_{n}^{\alpha, \beta}\left(t^{j} ; u\right) .
\end{aligned}
$$

Lemma 2.4 Let $e_{r}(t)=t^{r}$ for $r \in\{0,1,2,3,4\}$ be the test functions and $E_{n}(u)$ be defined in (2.1). Then

$$
\begin{aligned}
& M_{n}^{\alpha, \beta}\left(e_{0} ; u\right)=1, \\
& M_{n}^{\alpha, \beta}\left(e_{1} ; u\right)=\frac{n}{n+\beta} u+\frac{\lambda+\alpha+1}{n+\beta},
\end{aligned}
$$




$$
\begin{aligned}
M_{n}^{\alpha, \beta}\left(e_{2} ; u\right)= & \frac{n^{2}}{(n+\beta)^{2}} u^{2}+\left(4+2 \lambda+2 \alpha+2 i E_{n}(u)\right) \frac{n}{(n+\beta)^{2}} u \\
& +\frac{(\lambda+1)(\lambda+2 \alpha+2)+\alpha^{2}}{(n+\beta)^{2}}, \\
M_{n}^{\alpha, \beta}\left(e_{3} ; u\right)= & \frac{n^{3}}{(n+\beta)^{3}} u^{3}+\left(9+3 \alpha+3 \lambda-2 i E_{n}(u)\right) \frac{n^{2}}{(n+\beta)^{3}} u^{2} \\
& +\left(18+12 \alpha+3 \lambda(\lambda+5+2 \alpha)+3 \alpha^{2}+4 i^{2}+2 i(8+3 \lambda+3 \alpha) E_{n}(u)\right) \\
& \times \frac{n}{(n+\beta)^{3}} u+\frac{\lambda^{3}+\alpha^{3}+\lambda^{2}(6+3 \alpha)+\lambda\left(11+9 \alpha+3 \alpha^{2}\right)+6+6 \alpha+3 \alpha^{2}}{(n+\beta)^{3}}, \\
M_{n}^{\alpha, \beta}\left(e_{4} ; u\right)= & \frac{n^{4}}{(n+\beta)^{4}} u^{4}+\mathcal{O}\left(\frac{1}{n+\beta}\right) \text { for each compact subset of }[0, \infty) .
\end{aligned}
$$

Proof The proof follows immediately from Lemma 2.2.

Lemma 2.5 Let $\psi_{u}^{r}(t)=(t-u)^{r}, r \in \mathbb{N}$, be the central moments of $M_{n}^{\alpha, \beta}$. Then

$$
\begin{aligned}
M_{n}^{\alpha, \beta}\left(\psi_{u}^{0} ; u\right)= & 1, \\
M_{n}^{\alpha, \beta}\left(\psi_{u}^{1} ; u\right)= & \frac{1}{(n+\beta)}(1+\lambda+\alpha-\beta u), \\
M_{n}^{\alpha, \beta}\left(\psi_{u}^{2} ; u\right)= & \frac{\beta^{2}}{(n+\beta)^{2}} u^{2}+2\left(1+i E_{n}(u)-2 \beta \frac{1+\lambda+\alpha}{n}\right) \frac{n}{(n+\beta)^{2}} u \\
& +\frac{\alpha^{2}+(\lambda+1)(\lambda+2 \alpha+2)}{(n+\beta)^{2}}, \\
M_{n}^{\alpha, \beta}\left(\psi_{u}^{4} ; u\right)= & \mathcal{O}\left((n+\beta)^{-1}\right) \quad \text { for each compact subset of }[0, \infty) .
\end{aligned}
$$

Proof In the light of Lemmas 2.2 and 2.3, we can easily prove Lemma 2.5.

Definition 2.6 Let $g \in C[0, \infty)$. Then the modulus of continuity for a uniformly continuous function $g$ is defined as follows:

$$
\omega(g ; \delta)=\sup _{\left|t_{1}-t_{2}\right| \leq \delta}\left|g\left(t_{1}\right)-g\left(t_{2}\right)\right|, \quad t_{1}, t_{2} \in[0, \infty) .
$$

For a uniformly continuous function $g$ in $C[0, \infty)$ and $\delta>0$, we get

$$
\left|g\left(t_{1}\right)-g\left(t_{2}\right)\right| \leq\left(1+\frac{\left(t_{1}-t_{2}\right)^{2}}{\delta^{2}}\right) \omega(g ; \delta)
$$

Theorem 2.7 Let $M_{n}^{\alpha, \beta}$ be the operators defined by (1.5) and $g \in C[0, \infty) \cap\{g: u \geq$ $0, \frac{g(u)}{1+u^{2}}$ is convergent as $\left.u \rightarrow \infty\right\}$. Then the operators are defined by $(1.5), M_{n}^{\alpha, \beta} \rightrightarrows g$ on each compact subset of $[0, \infty)$, where $\rightrightarrows$ stands for uniform convergence.

Proof For the proof of uniformity for the operators $M_{n}^{\alpha, \beta}$, we use the well-known Korovkin theorem. So, it is sufficient to show that

$$
M_{n}^{\alpha, \beta}\left(e_{\nu} ; u\right) \rightarrow e_{\nu}(u) \quad \text { for } v=0,1,2 .
$$


Using Lemma 2.4, it is obvious that $M_{n}^{\alpha, \beta}\left(e_{v} ; u\right) \rightarrow e_{v}(u)$ as $n \rightarrow \infty, v=0,1,2$, which proves Theorem 2.7.

Theorem 2.8 (See [34]) Let $L: C([a, b]) \rightarrow B([a, b])$ be a linear and positive operator, and let $\varphi_{x}$ be the function defined by

$$
\varphi_{x}(t)=|t-x|, \quad(x, t) \in[a, b] \times[a, b] .
$$

Iff $\in C_{B}([a, b])$ for any $x \in[a, b]$ and any $\delta>0$, the operator $L$ verifies

$$
\begin{aligned}
|(L f)(x)-f(x)| \leq & |f(x)|\left|\left(L e_{0}\right)(x)-1\right| \\
& +\left\{\left(L e_{0}\right)(x)+\delta^{-1} \sqrt{\left(L e_{0}\right)(x)\left(L \varphi_{x}^{2}\right)(x)}\right\} \omega_{f}(\delta),
\end{aligned}
$$

where $C[a, b]$ is the space of all continuous functions defined on $[a, b]$, and $C_{B}[a, b]$ is the space of all continuous and bounded functions defined on $[a, b]$.

Theorem 2.9 For the operators $M_{n}^{\alpha, \beta}$ given by (1.5) and $g \in C_{B}[0, \infty)$, we have

$$
\left|M_{n}^{\alpha, \beta}(g ; u)-g(u)\right| \leq 2 \omega(g ; \delta),
$$

where $\delta=\sqrt{M_{n}^{\alpha, \beta}\left(\psi_{u}^{2} ; u\right)}$ and $C_{B}[0, \infty)$ is the space of continuous and bounded functions defined on $[0, \infty)$.

Proof Using Lemma 2.4, Lemma 2.5, and Theorem 2.8, we get

$$
\left|M_{n}^{\alpha, \beta}(g ; u)-g(u)\right| \leq\left\{1+\delta^{-1} \sqrt{M_{n}^{\alpha, \beta}\left(\psi_{u}^{2} ; u\right)}\right\} \omega(g ; \delta)
$$

On taking $\delta=\sqrt{M_{n}^{\alpha, \beta}\left(\psi_{u}^{2} ; u\right)}$, we arrive at the required result.

\section{Direct results}

Let us equip the norm on $g$ such as $\|g\|=\sup _{0 \leq u<\infty}|g(u)|$. For any $g \in C_{B}[0, \infty)$ and $\delta>0$, Peetre's K-functional is defined as follows:

$$
K_{2}(g, \delta)=\inf \left\{\|g-h\|+\delta\left\|h^{\prime \prime}\right\|: h \in C_{B}^{2}[0, \infty)\right\}
$$

where $C_{B}^{2}[0, \infty)=\left\{h \in C_{B}[0, \infty): h^{\prime}, h^{\prime \prime} \in C_{B}[0, \infty)\right\}$. From DeVore and Lorentz ([8], p.177, Theorem 2.4), there exists an absolute constant $C>0$ in such a way that

$$
K_{2}(g ; \delta) \leq C \omega_{2}(g ; \sqrt{\delta})
$$

Lemma 3.1 Let the auxiliary operators be

$$
\widehat{M}_{n}^{\alpha, \beta}(g ; u)=M_{n}^{\alpha, \beta}(g ; u)+g(u)-g\left(\frac{n}{n+\beta} u+\frac{\lambda+\alpha+1}{n+\beta}\right) .
$$


For $h, g \in C_{B}^{2}[0, \infty), u \geq 0$, and $\mu, \lambda \geq 0$, we get

$$
\left|\widehat{M}_{n}^{\alpha, \beta}(g ; u)-g(u)\right| \leq \xi_{n}^{u}\left\|h^{\prime \prime}\right\|,
$$

where

$$
\xi_{n}^{u}=M_{n}^{\alpha, \beta}\left(\psi_{u}^{2} ; u\right)+\left(M_{n}^{\alpha, \beta}\left(\psi_{u}^{1} ; u\right)\right)^{2} .
$$

Proof From (3.2), we have

$$
\widehat{M}_{n}^{\alpha, \beta}(1 ; u)=1, \quad \widehat{M}_{n}^{\alpha, \beta}\left(\psi_{u} ; u\right)=0 \quad \text { and } \quad\left|\widehat{M}_{n}^{\alpha, \beta}(g ; u)\right| \leq 3\|g\| .
$$

From Taylor's series for $h \in C_{B}^{2}[0, \infty)$, we have

$$
h(t)=h(u)+(t-u) h^{\prime}(u)+\int_{u}^{t}(t-v) h^{\prime \prime}(v) d v .
$$

Using $\widehat{M}_{n}^{\alpha, \beta}(g ; u)$ in (3.4), we get

$$
\widehat{M}_{n}^{\alpha, \beta}(h ; u)-h(u)=h^{\prime}(u) \widehat{M}_{n}^{\alpha, \beta}(t-u ; u)+\widehat{M}_{n}^{\alpha, \beta}\left(\int_{u}^{t}(t-v) h^{\prime \prime}(v) d v ; u\right) .
$$

From (3.2) and (3.3), we have

$$
\begin{aligned}
& \widehat{M}_{n}^{\alpha, \beta}(h ; u)-h(u)=\widehat{M}_{n}^{\alpha, \beta}\left(\int_{u}^{t}(t-v) h^{\prime \prime}(v) d v ; u\right) \\
& =M_{n}^{\alpha, \beta}\left(\int_{u}^{t}(t-v) h^{\prime \prime}(v) d v ; u\right) \\
& \quad-\int_{u}^{\frac{n}{n+\beta} u+\frac{\lambda+\alpha+1}{n+\beta}}\left(\frac{n}{n+\beta} u+\frac{\lambda+\alpha+1}{n+\beta}-v\right) g^{\prime \prime}(v) d v, \\
& \left|\widehat{M}_{n}^{\alpha, \beta}(h ; u)-h(u)\right| \\
& \leq\left|M_{n}^{\alpha, \beta}\left(\int_{u}^{t}(t-v) h^{\prime \prime}(v) d v ; u\right)\right| \\
& \quad+\left|\int_{u}^{\frac{n}{n+\beta} u+\frac{\lambda+\alpha+1}{n+\beta}}\left(\frac{n}{n+\beta} u+\frac{\lambda+\alpha+1}{n+\beta}-v\right) h^{\prime \prime}(v) d v\right| .
\end{aligned}
$$

Since

$$
\left|\int_{u}^{t}(t-v) h^{\prime \prime}(v) d v\right| \leq(t-v)^{2}\left\|h^{\prime \prime}\right\|
$$

we get

$$
\left|\int_{u}^{M_{n}^{\alpha, \beta}\left(e_{1} ; u\right)}\left(M_{n}^{\alpha, \beta}\left(e_{1} ; u\right)-v\right) h^{\prime \prime}(v) d v\right| \leq\left(M_{n}^{\alpha, \beta}(t-v ; u)\right)^{2}\left\|h^{\prime \prime}\right\| .
$$

Combining (3.5), (3.6), and (3.7), we have

$$
\widehat{M}_{n}^{\alpha, \beta}(h ; u)-h(u) \mid \leq \xi_{n}^{u}\left\|h^{\prime \prime}\right\| .
$$


Theorem 3.2 Let $f \in C_{B}^{2}[0, \infty)$. Then there exists a constant $C>0$ such that

$$
\left|M_{n}^{\alpha, \beta}(g ; u)-g(u)\right| \leq C \omega_{2}\left(g ; \frac{1}{2} \sqrt{\xi_{n}^{u}}\right)+\omega\left(g ; M_{n}^{\alpha, \beta}\left(\psi_{u} ; u\right),\right.
$$

where $\xi_{n}^{u}$ is defined in Lemma 3.1.

Proof For $h \in C_{B}^{2}[0, \infty)$ and $g \in C_{B}[0, \infty)$ and by the definition of $\widehat{M}_{n}^{\alpha, \beta}$, we have

$$
\begin{aligned}
\left|M_{n}^{\alpha, \beta}(g ; u)-g(u)\right| \leq & \left.\left|\widehat{M}_{n}^{\alpha, \beta}(g-h ; u)\right|+|(g-h)(u)|+\mid \widehat{M}_{n}^{\alpha, \beta}(h ; u)-h(u)\right) \mid \\
& +\left|g\left(\frac{n}{n+\beta} u+\frac{\lambda+\alpha+1}{n+\beta}\right)-g(u)\right| .
\end{aligned}
$$

From Lemma 3.1 and relations in (3.3), one gets

$$
\begin{aligned}
\left|M_{n}^{\alpha, \beta}(g ; u)-g(u)\right| \leq & 4\|g-h\|+\left|\widehat{M}_{n}^{\alpha, \beta}(h ; u)-h(u)\right| \\
& +\left|g\left(\frac{n}{n+\beta} u+\frac{\lambda+\alpha+1}{n+\beta}\right)-g(u)\right| \\
\leq & 4\|g-h\|+\xi_{n}^{u}\left\|h^{\prime \prime}\right\|+\omega\left(g ; M_{n}^{\alpha, \beta}\left(\psi_{u} ; u\right)\right) .
\end{aligned}
$$

Using the definition of Peetre's K-functional, we have

$$
\left|M_{n}^{\alpha, \beta}(g ; u)-g(u)\right| \leq C \omega_{2}\left(g ; \frac{1}{2} \sqrt{\xi_{n}^{u}}\right)+\omega\left(g ; M_{n}^{\alpha, \beta}\left(\psi_{u} ; u\right) .\right.
$$

This completes the proof of Theorem 3.2.

We consider the Lipschitz type space [30]:

$$
\operatorname{Lip}_{M}^{k_{1}, k_{2}}(\rho):=\left\{g \in C_{B}[0, \infty):|g(t)-g(u)| \leq M \frac{|t-u|^{\rho}}{\left(t+k_{1} u+k_{2} u^{2}\right)^{\frac{\rho}{2}}}: u, t \in(0, \infty)\right\}
$$

where $M \geq 0$ is a constant, $k_{1}, k_{2}>0, \rho>0$, and $\rho \in(0,1]$.

Theorem 3.3 For $g \in \operatorname{Lip}_{M}^{k_{1}, k_{2}}(\rho)$, we have

$$
\left|M_{n}^{\alpha, \beta}(g ; u)-g(u)\right| \leq M\left(\frac{\eta_{n}^{*}(u)}{k_{1} u+k_{2} u^{2}}\right)^{\frac{\rho}{2}}
$$

where $u>0$ and $\eta_{n}^{*}(u)=M_{n}^{\alpha, \beta}\left(\psi_{u}^{2} ; u\right)$.

Proof For $\rho=1$, we have

$$
\begin{aligned}
\left|M_{n}^{\alpha, \beta}(g ; u)-g(u)\right| & \leq M_{n}^{\alpha, \beta}(|g(t)-g(u)|)(u) \\
& \leq M M_{n}^{\alpha, \beta}\left(\frac{|t-u|}{\left(t+k_{1} u+k_{2} u^{2}\right)^{\frac{1}{2}}} ; u\right) .
\end{aligned}
$$


Since $\frac{1}{t+k_{1} u+k_{2} u^{2}}<\frac{1}{k_{1} u+k_{2} u^{2}}$ for all $t, u \in(0, \infty)$, we get

$$
\begin{aligned}
\left|M_{n}^{\alpha, \beta}(g ; u)-g(u)\right| & \leq \frac{M}{\left(k_{1} u+k_{2} u^{2}\right)^{\frac{1}{2}}}\left(M_{n}^{\alpha, \beta}\left((t-u)^{2} ; x\right)\right)^{\frac{1}{2}} \\
& \leq M\left(\frac{\eta_{n}^{*}(u)}{k_{1} u+k_{2} u^{2}}\right)^{\frac{1}{2}} .
\end{aligned}
$$

Thus Theorem 3.3 holds for $\rho=1$. Next, for $\rho \in(0,1)$ and from Hölder's inequality with $p=\frac{2}{\rho}$ and $q=\frac{2}{2-\rho}$, we get

$$
\begin{aligned}
\left|M_{n}^{\alpha, \beta}(g ; u)-g(u)\right| & \leq\left(M_{n}^{\alpha, \beta}\left((|g(t)-g(u)|)^{\frac{2}{\rho}} ; u\right)\right)^{\frac{\rho}{2}} \\
& \leq M\left(M_{n}^{\alpha, \beta}\left(\frac{|t-u|^{2}}{\left(t+k_{1} u+k_{2} u^{2}\right)} ; u\right)\right)^{\frac{\rho}{2}} .
\end{aligned}
$$

Since $\frac{1}{t+k_{1} u+k_{2} u^{2}}<\frac{1}{k_{1} u+k_{2} u^{2}}$ for all $t, u \in(0, \infty)$, we obtain

$$
\begin{aligned}
\left|M_{n}^{\alpha, \beta}(g ; u)-g(u)\right| & \leq M\left(\frac{M_{n}^{\alpha, \beta}\left(|t-u|^{2} ; u\right)}{k_{1} u+k_{2} u^{2}}\right)^{\frac{\rho}{2}} \\
& \leq M\left(\frac{\eta_{n}^{*}(u)}{k_{1} u+k_{2} u^{2}}\right)^{\frac{\rho}{2}} .
\end{aligned}
$$

Hence, we prove Theorem 3.3.

\section{Global approximation}

From [10], we recall some notation to prove the global approximation results.

For the weight function $1+u^{2}$ and $0 \leq u<\infty$, we have

$$
B_{1+u^{2}}[0, \infty)=\left\{f(u):|f(u)| \leq M_{f}\left(1+u^{2}\right)\right\}
$$

where $M_{f}$ is a constant depending on $f$ and $C_{1+u^{2}}[0, \infty) \subset B_{1+u^{2}}[0, \infty)$ is a space of continuous functions equipped with the norm $\|f\|_{1+u^{2}}=\sup _{u \in[0, \infty)} \frac{|f|}{1+u^{2}}$.

Denote

$$
C_{1+u^{2}}^{k}[0, \infty)=\left\{f \in C_{1+u^{2}}: \lim _{u \rightarrow \infty} \frac{f(u)}{1+u^{2}}=k \text {, where } k \text { is a constant }\right\} .
$$

Theorem 4.1 Let the operators be defined by (1.5) acting from $C_{1+u^{2}}^{k}[0, \infty)$ to $B_{1+u^{2}}[0, \infty)$. Then, for every $f \in C_{1+u^{2}}^{k}[0, \infty)$, we have

$$
\lim _{n \rightarrow \infty}\left\|M_{n}^{\alpha, \beta}(g ; u)-g\right\|_{1+u^{2}}=0
$$

where $B_{1+u^{2}}$ and $C_{1+u^{2}}^{k}$ are defined in (4.1) and (4.2).

Proof To prove this result, it is sufficient to show that

$$
\lim _{n \rightarrow \infty}\left\|M_{n}^{\alpha, \beta}\left(e_{i} ; u\right)-u^{i}\right\|_{1+u^{2}}=0, \quad i=0,1,2
$$


Using Lemma 2.4, we obtain

$$
\left\|M_{n}^{\alpha, \beta}\left(e_{0} ; u\right)-u^{0}\right\|_{1+u^{2}}=\sup _{u \in[0, \infty)} \frac{\left|M_{n}^{\alpha, \beta}(1 ; u)-1\right|}{1+u^{2}}=0 \quad \text { for } i=0 .
$$

For $i=1$,

$$
\begin{aligned}
\left\|M_{n}^{\alpha, \beta}\left(e_{1} ; u\right)-u\right\|_{1+u^{2}} & =\sup _{u \in[0, \infty)} \frac{\left(\frac{n}{n+\beta}-1\right) u+\frac{\lambda+\alpha+1}{n+\beta}}{1+u^{2}} \\
& =\left(\frac{n}{n+\beta}-1\right) \sup _{u \in[0, \infty)} \frac{u}{1+u^{2}}+\frac{\lambda+\alpha+1}{n+\beta} \sup _{u \in[0, \infty)} \frac{1}{1+u^{2}} .
\end{aligned}
$$

This shows that $\left\|M_{n}^{\alpha, \beta}\left(e_{1} ; u\right)-u\right\|_{1+u^{2}} \rightarrow 0, n \rightarrow \infty$. For $i=2$,

$$
\begin{aligned}
\left\|M_{n}^{\alpha, \beta}\left(e_{2} ; u\right)-u^{2}\right\|_{1+u^{2}}= & \sup _{u \in[0, \infty)} \frac{\left|M_{n}^{\alpha, \beta}\left(e_{2} ; u\right)-u^{2}\right|}{1+u^{2}} \\
\leq & \left(\frac{n^{2}}{(n+\beta)^{2}}-1\right) \sup _{u \in[0, \infty)} \frac{u^{2}}{1+u^{2}} \\
& +\left(4+2 \lambda+2 \alpha+2 i E_{n}(u)\right) \frac{n}{(n+\beta)^{2}} \sup _{u \in[0, \infty)} \frac{u}{1+u^{2}} \\
& +\frac{(\lambda+1)(\lambda+2 \alpha+2)+\alpha^{2}}{(n+\beta)^{2}} \sup _{u \in[0, \infty)} \frac{1}{1+u^{2}},
\end{aligned}
$$

which shows that $\left\|M_{n}^{\alpha, \beta}\left(e_{2} ; u\right)-u^{2}\right\|_{1+u^{2}} \rightarrow 0, n \rightarrow \infty$.

Let $f \in C_{\rho}^{k}[0, \infty)$, Yüksel and Ispir [41] introduced the weighted modulus of continuity as follows:

$$
\Omega(g ; \delta)=\sup _{u \in[0, \infty), 0<h \leq \delta} \frac{|g(u+h)-g(u)|}{1+(u+h)^{2}} .
$$

Lemma 4.2 ([41]) Let $f \in C_{1+x^{2}}^{k}[0, \infty)$. Then

(i) $\Omega(f ; \delta)$ is a monotone increasing function of $\delta$;

(ii) $\lim _{\delta \rightarrow 0^{+}} \Omega(f ; \delta)=0$;

(iii) for each $\lambda \in[0, \infty), \Omega(f ; \lambda \delta) \leq(1+\lambda)\left(1+\delta^{2}\right) \Omega(f ; \delta)$.

For $t, x \in[0, \infty)$, one gets

$$
|f(t)-f(x)| \leq 2\left(1+\frac{|t-x|}{\delta}\right)\left(1+\delta^{2}\right)\left(1+x^{2}\right)\left(1+(t-x)^{2}\right) \Omega(f ; \delta) .
$$

Theorem 4.3 Let $g \in C_{1+u^{2}}^{k}[0, \infty)$. Then we have

$$
\sup _{u \in[0, \infty)} \frac{\left|M_{n}^{\alpha, \beta}(g ; u)-g(x)\right|}{\left(1+u^{2}\right)^{3}} \leq C(n)\left(1+\frac{1}{n+\beta}\right) \Omega\left(g ; \frac{1}{\sqrt{n+\beta}}\right)
$$

where $C(n)=1+\frac{C_{1}}{n+\beta} \cdot \frac{3 \sqrt{(3)}}{16}+\frac{\sqrt{C_{1}}}{2}+\frac{1}{4} \sqrt{\frac{C_{1} C_{2}}{n+\beta}}$ and $C_{1}, C_{2} \in(0, \infty)$. 
Proof Using (4.3) and $x, t \in(0, \infty)$, we have

$$
\begin{aligned}
\left|M_{n}^{\alpha, \beta}(g ; u)-g(u)\right| \leq & 2\left(1+\frac{M_{n}^{\alpha, \beta}(|t-u| ; u)}{\delta}\right)\left(1+\delta^{2}\right)\left(1+u^{2}\right) \\
& \times\left(1+M_{n}^{\alpha, \beta}\left((t-u)^{2} ; u\right)\right) \Omega(g ; \delta) .
\end{aligned}
$$

Applying the Cauchy-Schwarz inequality for (4.4), we get

$$
\begin{aligned}
& \left|M_{n}^{\alpha, \beta}(g ; u)-g(u)\right| \\
& \quad \leq 2\left(1+\delta^{2}\right)\left(1+u^{2}\right) \Omega(g ; \delta)\left(1+M_{n}^{\alpha, \beta}\left((t-u)^{2} ; u\right)\right. \\
& \left.\quad+\frac{\sqrt{M_{n}^{\alpha, \beta}\left((t-u)^{2} ; u\right)}}{\delta}+\frac{\sqrt{M_{n}^{\alpha, \beta}\left((t-u)^{2} ; u\right) M_{n}^{\alpha, \beta}\left((t-u)^{4} ; u\right)}}{\delta}\right) .
\end{aligned}
$$

By elementary calculations, we get

$$
\begin{aligned}
& \lim _{n \rightarrow \infty}(n+\beta) M_{n}^{\alpha, \beta}\left(\psi_{u}^{2} ; u\right)=2(1+i) u \\
& \lim _{n \rightarrow \infty}(n+\beta) M_{n}^{\alpha, \beta}\left(\psi_{u}^{4} ; u\right)=3(8 i+1) u^{3} .
\end{aligned}
$$

It follows that, for each fixed point $u>0$, there exists $N_{u} \in \mathbb{N}$ such that, for all $n>N_{u}$,

$$
\begin{aligned}
& M_{n}^{\alpha, \beta}\left(\psi^{2} ; u\right) \leq C_{1} \frac{u}{n+\beta}, \\
& M_{n}^{\alpha, \beta}\left(\psi^{4} ; u\right) \leq C_{2} \frac{u^{3}}{n+\beta},
\end{aligned}
$$

where $C_{1}, C_{2} \in(0, \infty)$. From (4.5)-(4.7) and choosing $\delta=\frac{1}{\sqrt{n+\beta}}$, we get the required result.

\section{A-statistical convergence}

From $[7,15]$ we first introduce convergence approximation theorems in operators theory. Here, we recall same notation from $[7,15]$. Let $A=\left(a_{n k}\right)$ be a nonnegative infinite summability matrix. For a given sequence $x:=\left(x_{k}\right)$, the $A$-transform of $x$ denoted by $A x:\left((A x)_{n}\right)$ is defined as follows:

$$
(A x)_{n}=\sum_{k=1}^{\infty} a_{n k} x_{k}
$$

provided the series converges for each $n$. A is said to be regular if $\lim (A x)_{n}=L$ whenever $\lim x=L$. Then $x=\left(x_{n}\right)$ is said to be an $A$-statistical convergence to $L$ i.e. $s t_{A}-\lim x=L$ if, for every $\epsilon>0, \lim _{n} \sum_{k:\left|x_{k}-L\right| \geq \epsilon} a_{n k}=0$. In addition, A-statistical convergence results are studied in $[13,22,23]$.

Theorem 5.1 Let $A=\left(a_{n k}\right)$ be a nonnegative regular summability matrix and $x \geq 0$. Then we have

$$
s t_{A}-\lim _{n}\left\|M_{n}^{\alpha, \beta}(g ; u)-g\right\|_{1+u^{2}}=0 \quad \text { for all } g \in C_{1+u^{2}}^{k}[0, \infty) \text {. }
$$


Proof From ([9], p. 191, Th. 3), it is sufficient to show that, for $\lambda_{1}=0$,

$$
s t_{A}-\lim _{n}\left\|M_{n}^{\alpha, \beta}\left(e_{i} ; u\right)-e_{i}\right\|_{1+u^{2}}=0 \quad \text { for } i \in\{0,1,2\} .
$$

Using Lemma 2.4, we have

$$
\begin{aligned}
\left\|M_{n}^{\alpha, \beta}\left(e_{1} ; u\right)-u\right\|_{1+u^{2}} & =\sup _{u \in[0, \infty)} \frac{1}{1+u^{2}}\left|\left(\frac{n}{n+\beta}-1\right) u+\frac{\lambda+\alpha+1}{n+\beta}\right| \\
& \leq\left|\frac{n}{n+\beta}-1\right| \sup _{u \in[0, \infty)} \frac{u}{1+u^{2}}+\left|\frac{\lambda+\alpha+1}{n+\beta}\right| \sup _{u \in[0, \infty)} \frac{1}{1+u^{2}} \\
& \leq \frac{1}{2}\left|\frac{n}{n+\beta}-1\right|+\left|\frac{\lambda+\alpha+1}{n+\beta}\right|
\end{aligned}
$$

We have

$$
s t_{A}-\lim _{n} \frac{1}{2}\left|\frac{n}{n+\beta}-1\right|=s t_{A}-\lim _{n}\left|\frac{\lambda+\alpha+1}{n+\beta}\right|=0 .
$$

Now, for given $\epsilon>0$, we define the following sets:

$$
\begin{aligned}
& J_{1}:=\left\{n:\left\|M_{n}^{\alpha, \beta}\left(e_{1} ; u\right)-u\right\| \geq \epsilon\right\}, \\
& J_{2}:=\left\{n: \frac{1}{2}\left|\frac{n}{n+\beta}-1\right| \geq \frac{\epsilon}{2}\right\}, \\
& J_{3}:=\left\{n:\left|\frac{\lambda+\alpha+1}{n+\beta}\right| \geq \frac{\epsilon}{2}\right\} .
\end{aligned}
$$

This implies that $J_{1} \subseteq J_{2} \cup J_{3}$, which shows that $\sum_{k_{1} \in J_{1}} a_{n k_{1}} \leq \sum_{k_{1} \in J_{2}} a_{n k}+\sum_{k_{1} \in J_{3}} a_{n k}$. Hence, from (5.2) we get

$$
s t_{A}-\lim _{n}\left\|M_{n}^{\alpha, \beta}\left(e_{1} ; u\right)-u\right\|_{1+u^{2}}=0 .
$$

In a similar way the following can be proved:

$$
s t_{A}-\lim _{n}\left\|M_{n}^{\alpha, \beta}\left(e_{2} ; u\right)-u^{2}\right\|_{1+u^{2}}=0 .
$$

This completes the proof of Theorem 5.1.

\section{Conclusion}

The motive of the present paper is to give a generalized error estimation of convergence by modification of Szász-Mirakyan integral operators via Dunkl analogue. We have defined the Szász-Mirakyan integral operators based on Dunkl analogue with the aid of two nonnegative parameters $0 \leq \alpha \leq \beta$. This type of modification enables us to give a generalied error estimation for a certain function in comparison to the Szász-Mirakyan integral operators based on Dunkl analogue. We investigated some approximation results by means of the well-known Korovkin type theorem. We have also calculated the rate of convergence of operators by means of Peetre's K-functional and second order modulus of continuity. Lastly, we studied the global approximation results. 


\section{Acknowledgements}

All authors are extremely grateful to the reviewers for their valuable suggestions and their crucial role in leading to a better presentation of this manuscript.

Funding

Not applicable.

\section{Availability of data and materials}

Not applicable.

\section{Competing interests}

All authors are grateful for this manuscript and declare that they have no competing interests.

\section{Authors' contributions}

All ideas of this paper was equally contributed by authors. All authors have agreed to the integrity and accuracy of this manuscript. All authors read and approved the final manuscript.

\section{Author details}

1Department of Mathematics, Faculty of Science, University of Tabuk, Tabuk, Saudi Arabia. ${ }^{2}$ Department of Mathematics, Jamia Millia Islamia, New Delhi, India. ${ }^{3}$ Faculty of Applied Sciences, Dr APJ Abdul Kalam Technical University, Lucknow, India. ${ }^{4}$ Post Graduate Department of Mathematics, Patna University, Bihar, India.

\section{Publisher's Note}

Springer Nature remains neutral with regard to jurisdictional claims in published maps and institutional affiliations.

Received: 25 November 2019 Accepted: 13 February 2020 Published online: 22 February 2020

\section{References}

1. Acar, T., Aral, A., Mohiuddine, S.A.: Approximation by bivariate $(p, q)$-Bernstein Kantorovich operators. Iran. J. Sci. Technol., Trans. A, Sci. 42(2), 655-662 (2018)

2. Acar, T., Aral, A., Mursaleen, M.: Approximation by Baskakov-Durrmeyer operators based on $(p, q)$-integers. Math. Slovaca 68(4), 897-906 (2018)

3. Acar, T., Aral, A., Raşa, I.: Positive linear operators preserving $\tau$ and $\tau^{2}$. Constr. Math. Anal. 2(3), 98-102 (2019)

4. Acar, T., Mohiuddine, S.A., Mursaleen, M.: Approximation by $(p, q)$-Baskakov-Durrmeyer-Stancu operators. Complex Anal. Oper. Theory 12(6), 1453-1468 (2018)

5. Acar, T., Mursaleen, M., Mohiuddine, S.A.: Stancu type $(p, q)$-Szász-Mirakyan-Baskakov operators. Commun. Fac. Sci. Univ. Ank. Sér. A1 67(1), 116-128 (2018)

6. Alotaibi, A., Nasiruzzaman, M., Mursaleen, M.: A Dunkl type generalization of Szász operators via post-quantum calculus. J. Inequal. Appl. 2018, Article ID 287 (2018)

7. Connor, J.S.: On strong matrix summability with respect to a modulus and statistical convergence. Can. Math. Bull. 32, 194-198 (1989)

8. DeVore, R.A., Lorentz, G.G.: Constructive Approximation. Grudlehren der Mathematischen Wissenschaften. Springer, Berlin (1993)

9. Duman, O., Orhan, C.: Statistical approximation by positive linear operators. Studio Math. 16(2), 187-197 (2004)

10. Gadziev, A.D.: Theorems of the type of P.P. Korovkin's theorems. Mat. Zametki 20(5), 781-786 (1976) (in Russian) (1976), Math. Notes 20(5-6), 995-998 (Engl. Trans.) (1976)

11. İçöz, G., Çekim, B.: Dunkl generalization of Szász operators via q-calculus. J. Inequal. Appl. 284, Article ID 11 (2015)

12. Iç̧öz, G., Çekim, B.: Stancu-type generalization of Dunkl analogue of Szász-Kantorovich operators. Math. Methods Appl. Sci. 39, 7 (2016). https://doi.org/10.1002/mma.3602

13. Kadak, U., Mohiuddine, S.A.: Generalized statistically almost convergence based on the difference operator which includes the $(p, q)$-gamma function and related approximation theorems. Results Math. 73(1), Article ID 9 (2018)

14. Kajla, A., Acar, T.: A new modification of Durrmeyer type mixed hybrid operators. Carpath. J. Math. 34(1), 47-56 (2018)

15. Kolk, E.: Matrix summability of statistically convergent sequences. Analysis 13, 77-83 (1993)

16. Krech, G., Krech, I.: On some bivariate Gauss-Weierstrass operators. Constr. Math. Anal. 2(2), 57-63 (2019)

17. Mazhar, S.M., Totik, V.: Approximation by modified Szász operators. Acta Sci. Math. 49(1-4), 257-269 (1985)

18. Milovanovic, G.V., Mursaleen, M., Nasiruzzaman, M.: Modified Stancu type Dunkl generalization of Szász-Kantorovich operators. Rev. R. Acad. Cienc. Exactas Fís. Nat., Ser. A Mat. 112, 135-151 (2018)

19. Mohiuddine, S.A., Acar, T, Alghamdi, M.A.: Genuine modified Bernstein-Durrmeyer operators. J. Inequal. Appl. 2018, Article ID 104 (2018)

20. Mohiuddine, S.A., Acar, T., Alotaibi, A.: Construction of a new family of Bernstein-Kantorovich operators. Math Methods Appl. Sci. 40, 7749-7759 (2017)

21. Mohiuddine, S.A., Acar, T., Alotaibi, A.: Durrmeyer type $(p, q)$-Baskakov operators preserving linear functions. J. Math. Inequal. 12, 961-973 (2018)

22. Mohiuddine, S.A., Alamri, B.A.S.: Generalization of equi-statistical convergence via weighted lacunary sequence with associated Korovkin and Voronovskaya type approximation theorems. Rev. R. Acad. Cienc. Exactas Fís. Nat., Ser. A Mat. 113(3), 1955-1973 (2019)

23. Mohiuddine, S.A., Asiri, A., Hazarika, B.: Weighted statistical convergence through di fference operator of sequences of fuzzy numbers with application to fuzzy approximation theorems. Int. J. Gen. Syst. 48(5), 492-506 (2019)

24. Mohiuddine, S.A., Hazarika, B., Alghamdi, M.A.: Ideal relatively uniform convergence with Korovkin and Voronovskaya types approximation theorems. Filomat 33(14), 4549-4560 (2019) 
25. Mohiuddine, S.A., Özger, F.: Approximation of functions by Stancu variant of Bernstein-Kantorovich operators based on shape parameter $\alpha$. Rev. R. Acad. Cienc. Exactas Fís. Nat., Ser. A Mat. 114, Article ID 70 (2020)

26. Mursaleen, M., Nasiruzzaman, M.: Dunkl generalization of Kantorovich type Szasz-Mirakjan operators via q- calculus. Asian-Eur. J. Math. 10(4), Article ID 1750077 (2017)

27. Mursaleen, M., Nasiruzzaman, M.: Approximation of modified Jakimovski-Leviatan-beta type operators. Constr. Math. Anal. 1(2), 88-98 (2018)

28. Mursaleen, M., Nasiruzzaman, M., Alotaibi, A.: On modified Dunkl generalization of Szasz operators via q-calculus. J. Inequal. Appl. 2018, Article ID 38 (2017)

29. Nasiruzzaman, M., Rao, N.: A generalized Dunkl type modifications of Phillips-operators. J. Inequal. Appl. 2018, Article ID 323 (2018)

30. Özarslan, M.A., Aktuğlu, H.: Local approximation for certain King type operators. Filomat 27, 173-181 (2013)

31. Ozsarac, F., Acar, T.: Reconstruction of Baskakov operators preserving some exponential functions. Math. Methods Appl. Sci. 42(16), 5124-5132 (2019)

32. Rao, N., Wafi, A., Acu, A.M.: q-Szász-Durrmeyer type operators based on Dunkl analogue. Complex Anal. Oper. Theory 13(3), 915-934 (2019)

33. Rosenblum, M.: Generalized Hermite polynomials and the Bose-like oscillator calculus. Oper. Theory, Adv. Appl. 73, 369-396 (1994)

34. Shisha, O., Bond, B.: The degree of convergence of sequences of linear positive operators. Proc. Natl. Acad. Sci. USA 60, 1196-1200 (1968)

35. Srivastava, H.M., Özger, F., Mohiuddine, S.A.: Construction of Stancu-type Bernstein operators based on Bézier bases with shape parameter $\lambda$. Symmetry $11(3)$, Article ID 316 (2019)

36. Sucu, S.: Dunkl analogue of Szász operators. Appl. Math. Comput. 244, 42-48 (2014)

37. Szász, O.: Generalization of Bernstein's polynomials to the infinite interval. J. Res. Natl. Bur. Stand. 45, 239-245 (1950)

38. Wafi, A., Rao, N.: A generalization of Szász-type operators which preserves constant and quadratic test functions. Cogent Math. 3, Article ID 1227023 (2016)

39. Wafi, A., Rao, N.: Szász-Durrmeyer operators based on Dunkl analogue. Complex Anal. Oper. Theory 12(7), 1519-1536 (2018)

40. Wafi, A., Rao, N.: Szasz-Gamma operators based on Dunkl analogue. Iran. J. Sci. Technol., Trans. A, Sci. 43(1), 213-223 (2019)

41. Yüksel, I., Ispir, N.: Weighted approximation by a certain family of summation integral-type operators. Comput. Math Appl. 52(10-11), 1463-1470 (2006)

\section{Submit your manuscript to a SpringerOpen ${ }^{\circ}$ journal and benefit from:}

- Convenient online submission

- Rigorous peer review

- Open access: articles freely available online

- High visibility within the field

- Retaining the copyright to your article

Submit your next manuscript at $>$ springeropen.com 